\title{
MPM: a hierarchical clustering algorithm using matrix partitioning method for non-numeric data
}

\author{
Hewijin Christine Jiau • Yi-Jen Su • Yeou-Min Lin • \\ Shang-Rong Tsai
}

(C) Springer Science + Business Media, LLC 2006

\begin{abstract}
The Publisher regrets that the original article incorrectly listed the authors' location as the "People's Republic of China". This was a typesetting error. Their correct location is the "Republic of China", which is also known as Taiwan. Their full affiliation is Department of Electrical Engineering, National Cheng Kung University, Tainan, Taiwan.
\end{abstract}

The online version of the original article can be found at http://dx.doi.org/10.1007/s10844-006-0250-2.

H. C. Jiau $\cdot$ Y.-J. Su $(\square) \cdot$ Y.-M. Lin · S.-R. Tsai

Department of Electrical Engineering, National Cheng Kung University,

Tainan, Taiwan

e-mail: iansu@ee.ncku.edu.tw

H. C. Jiau

e-mail: jiauhjc@mail.ncku.edu.tw

Y.-M. Lin

e-mail: base@dcl.ee.ncku.edu.tw

S.-R. Tsai

e-mail: srtsai@mail.ncku.edu.tw 